\title{
Evolution of Compression Processes in Aero-Engine Thermal Cycles
}

\author{
Y. Daren, T. Jingfeng ${ }^{*}$ and B. Wen \\ School of Energy Science and Engineering, Harbin Institute of Technology, Harbin 150001, China
}

\begin{abstract}
In order to provide a basis for the establishment of an applicable strategy for the development of aero-engine thermal cycles, the evolution of compression processes in aero-engine thermal cycles is reviewed in this paper by analyzing the compression requirements for the injection or extraction of energy. It is therefore concluded that the injection of energy in the compression is required for applications in low speed ranges, for example, the injection of energy in turbojet compressors; the method of adiabatic compression is desirable for applications in intermediate speed ranges, for example, shock interactions for ramjets/scramjets; and the extraction of energy is needed for applications in high speed ranges, for example, the extraction of energy in ramjets with energy-bypass. The injection or extraction of energy in the compression heavily depends on the aero-engine performance required for applications in different speed ranges.
\end{abstract}

Keywords: Aero-engine thermal cycle, compression process, energy injection or extraction, turbojet, ramjet/scramjet, ramjet with energy bypass.

\section{INTRODUCTION}

As the flight speed advanced from subsonic to supersonic, and even hypersonic, significant changes took place in aero-engine thermal cycles, especially in their compression processes. Theoretically, maximum overall engine efficiency depends on the maximum amount of work which can be added in the compression process of a turbojet (Riggins JPP 2004) [1]. Addition of work is usually possible in a compressor designed with overall engine efficiency taken into consideration. However, the permissible amount of work which can be added in the compression process decreases with the increasing flight Mach number, because the thermal limit of material at the combustor exit sets an upper limit on airflow total temperature. In addition, the use of a mechanical compressor is no longer needed for the compression at a higher speed, as airflow stagnation increases significantly with the increasing flight Mach number. Consequently, a ramjet without a work interaction device becomes a preferred choice for applications with the flight speed, for example, the Mach number $M a>3$. As it becomes more and more difficult to achieve the airflow compression required for subsonic combustion in the ramjet, especially for hypersonic applications, a supersonic combustion ramjet (scramjet), in which the supersonic combustion reduces the airflow compression, become a preferred choice for hypersonic applications.

When the flight speed increase further, for example, $M a$ $>9$, there are inevitably some technological challenges to the ramjet/scramjet compression. In order to ensure sufficient air/fuel mixing and stable combustion, the Mach number at the combustor inlet must be kept in a permissible range. In a hypersonic case, the compression of inlet airflow must be sufficient enough to satisfy this Mach number limit at the cost of an unacceptable compression loss and a significant

*Address correspondence to this author at the School of Energy Science and Engineering, Harbin Institute of Technology, Harbin 150001, China;

E-mail: tangjingfeng@hcms.hit.edu.cn increase in the static temperature at the compression exit, which deteriorate the performance of ramjet/scramjet. If the compression is mitigated, the Mach number at the combustor inlet will be too high to achieve sufficient air/fuel mixing and stable combustion over a practical combustor length. Therefore, a ramjet with energy-bypass is proposed as a solution of above mentioned problems (Fraishtadt Tech Phys 1998) [2], and its fundamental idea is that part of flow energy is extracted from the airflow field upstream of the combustor and then returned downstream of the combustor. The combination of an upstream energy extraction and a downstream energy return process is the so-called energy-bypass. The incoming airflow goes through the process of compression, energy extraction, combustion, energy return and expansion in such an engine. Depending on the upstream energy extraction, the compression is kept under a certain level with an acceptable process loss, and the airflow field in front of the combustor can be controlled to ensure a permissible Mach number at the combustor inlet. With the downstream energy injection, the efficient use of energy is achieved. Such operations improve the compression, combustion and eventually engine performance.

The ramjet with energy-bypass can be realized with the help of magneto-hydrodynamics (MHD) devices (Sheikin Tech Phys 1992) [3] (Sheikin Tech Phys 1993) [4]. In a MHD device, the axial body forces on flow elements are induced by the perpendicular electric and magnetic fields existing in the transverse plane. Deceleration or acceleration of airflow is caused by these body forces with electrical power generated or consumed onboard. Such an engine was first proposed under the name of AJAX (Kuranov AIAA 2001) [5] (Gurijanov AIAA 1996) [6], and another was studied under the name of MHD-Arc-Ramjet in which an arc heating device was used to return energy (Tang AIAA 2006) [7] (Tang AIAA 2007) [8]. In order to make MHD interactions effective, the appreciable electric conductivity of airflow is achieved using equilibrium ionization technologies at high enough flow temperatures (Park JPP 2001) [9], or nonequilibrium ionization technologies at medium flow tem- 
peratures (Sergey AIAA J 2001 ) [10] (Sergey JPP 2002) [11] (Park JPP 2003) [12]. The characteristics of the ramjet with energy-bypass is shown in Fig. (1) (seeing (Tang AIAA 2006) [7] for further details). The performance analyses in previous work have demonstrated that the performance of the ramjet with energy-bypass is better than ramjet/scramjet, especially for the flight with $M a>9$, and such an engine expands the scope of application in a higher flight Mach number. (Park JPP 2001) [9] (Park JPP 2003) [12] (Litchford JPP 2001) [13] (Park JPP 2001) [14] (Burakhanov JPP 2001) [15] (Kuranov JSR 2003) [16] (Heiser JPP 2005) [17] (Sheikin AIAA 2005) [18]

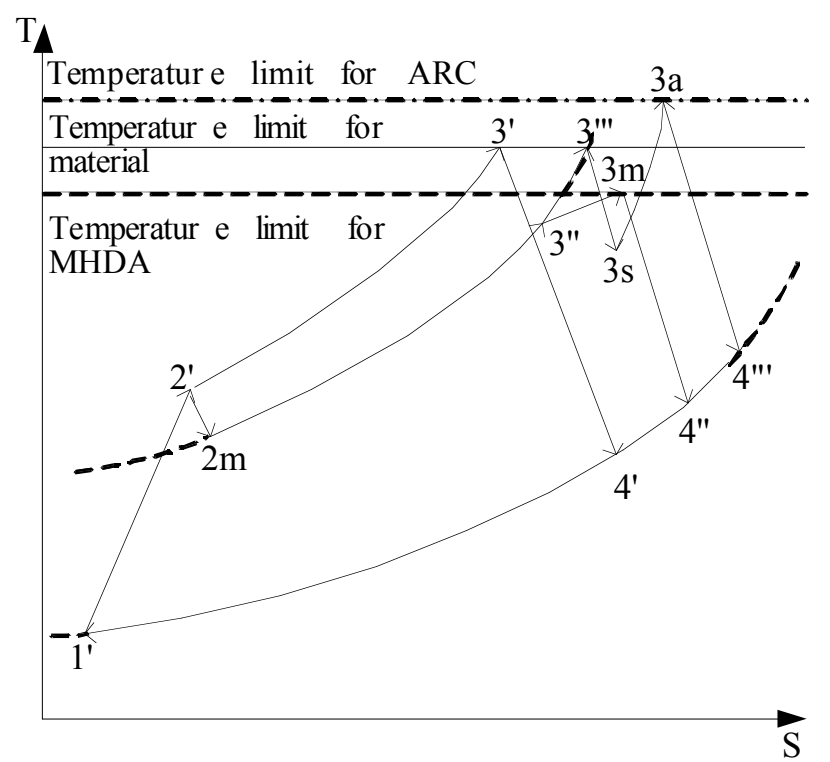

Fig. (1). Temperature-entropy cycle diagram of ramjets (1'-2'-3'-4'1': conventional ramjet; 1'-2'-2m-3"-3m-4"-1': MHD accelerator case; $1^{\prime}-2^{\prime}-2 \mathrm{~m}-3$ '"-3s-3a-4"'-1': ARC case; process $2^{\prime} \rightarrow 2 \mathrm{~m}$ : energy extraction; process $3^{\prime \prime} \rightarrow 3 \mathrm{~m}$ and $3 \mathrm{~s} \rightarrow 3 \mathrm{a}$ : energy injection).

To sum up, the turbojet cycle based on a mechanical compression with energy injected and a mechanical expansion with energy extracted, is applicable to applications with $M a=0.5 \sim 3$. The ramjet /scramjet cycle without the injection or extraction of energy in the compression, is suitable for applications with $M a=3 \sim 9$. The cycle of the ramjet with energy-bypass is a variant of Brayton thermal cycle for applications with $M a>9$, in which part of flow energy is extracted from the airflow field upstream of the combustor and then returned downstream of the combustor. So, it is worthwhile to discuss when the injection or exaction of energy is necessary in the compression process of an aeroengine thermal cycle, because it is believed that this discussion can leads to a good understanding of the development trend of aero-engine thermal cycles so that an applicable strategy can be established for the development of aeroengine thermal cycles.

The evolution of compression processes in aero-engine thermal cycles is reviewed in this paper by analyzing the compression requirements for the injection or extraction of energy.

\section{COMPRESSION REQUIREMENTS FOR INJECTION OR EXTRACTION OF ENERGY}

An ideal Brayton thermal cycle is made up of an isentropic compression process $(1 \rightarrow 2)$, an isobaric combustion process $(2 \rightarrow 3)$ and an isentropic expansion process $(3 \rightarrow 4)$. According to the aero-thermodynamic theory, the specific thrust of a Brayton cycle engine can be expressed as,

$$
F_{s p}=v_{4}-v_{1}=\sqrt{2 L_{i d}+v_{1}^{2}}-v_{1}
$$

where $F_{s p}$ is the specific thrust of engine; $L_{i d}$ is the specific work provided by the ideal Brayton cycle; and $v$ is the flow speed.

Based on the thermodynamic principle, the following can be easily derived,

$$
L_{i d}=C_{p} T_{1}\left(\pi^{\frac{\gamma-1}{\gamma}}-1\right)\left(\frac{\Delta}{\pi^{\frac{\gamma-1}{\gamma}}}-1\right)
$$

where $\pi=p_{t 2} / p_{1}$ is the pressure ratio; $\Delta=T_{t 3} / T_{1}$ is the temperature ratio; $C_{p}$ is the constant pressure specific heat; $\gamma$ is the specific heat ratio; and $p, p_{t}, T, T_{t}$ are the static pressure, total pressure, static temperature and total temperature respectively.

From equation 2:

(A1) The influence of factor $\pi=p_{t 2} / p_{1}(\pi \in[1,+\infty))$ : when $\pi=1, L_{i d}=0$, which can't occur at zero of cycle thermal efficiency; when $\pi=\pi_{\max }, L_{i d}=0$, which means the temperature at the compression exit reaches the limit imposed by the type of material and cooling methods, and the injection of energy is impossible in the combustion process. So $L_{i d}$ approaches its maximum when $\pi_{o p}=\left(\frac{T_{t 3}}{T_{1}}\right)^{\frac{\gamma}{2(\gamma-1)}}$.

(A2) The influence of factor $\Delta=T_{t 3} / T_{1}: L_{i d}$ benefits from the increase of $\Delta$, as $T_{t 3}$ increases at $\Delta$ under a fixed $T_{1}$, and $L_{i d}$ is improved as more heat is injected for a larger $T_{t 3}$.

From equations 1 and 2, it can be known that $F_{s p}$ benefits from the increase of $\Delta$, and $F_{s p}$ reaches its maximum in the case of $\pi_{o p}$.

For the design of engine, the type of material and cooling methods determine the value of $\Delta$ and the corresponding value of $\pi_{o p}$. For the function of engine, factor $\pi$ can be divided into two parts using expression $\pi=\frac{p_{t 2}}{p_{t 1}} \frac{p_{t 1}}{p_{1}}$. One part 
$\frac{p_{t 1}}{p_{1}}=\left(1+\frac{k-1}{2} M a_{1}^{2}\right)^{\frac{k}{k-1}}$ is about the characteristics of incoming airflow, and the other part $p_{t 2} / p_{t 1}$ is related to the compression mechanism. Under different incoming flow conditions, i.e. different $\frac{p_{t 1}}{p_{1}}$ values, factor $p_{t 2} / p_{t 1}$ must be adjusted to make $\pi$ approach $\pi_{o p}$ for the optimal gain of engine specific thrust. The degree of the compression required can be measured in terms of factor $p_{t 2} / p_{t 1}$. Qualitatively a larger value of $p_{t 2} / p_{t 1}$ is needed in the case of a less $p_{t 1} / p_{1}$, and a less value of $p_{t 2} / p_{t 1}$ is desirable in the case of a larger $p_{t 1} / p_{1}$.

It is known that the value of factor $p_{t 2} / p_{t 1}$ is related to the change in total pressure experienced in the compression process. With the help of Gibbs equation (Bejan Wiley 1988) [19], the definitions of total pressure and total temperature, the change in total pressure in the compression process can be expressed as,

$$
\begin{aligned}
\mathrm{d} s_{i r r} & =C_{p} \cdot \mathrm{d}(\ln T)-R \cdot \mathrm{d}(\ln p) \\
& =C_{p} \cdot \mathrm{d}\left(\ln T_{t}\right)-R \cdot \mathrm{d}\left(\ln p_{t}\right)
\end{aligned}
$$

where $\mathrm{d} s_{i r}$ is the differential of entropy; $R$ is the gas constant; and $\mathrm{d}(\ln p), \mathrm{d}\left(\ln p_{t}\right), \mathrm{d}(\ln T), \mathrm{d}\left(\ln T_{t}\right)$ are the differentials of corresponding factors.

From equation 3, the anticipated change in total pressure in the compression process, i.e. the needed $p_{t 2} / p_{t 1}$, can be achieved as the following indications,
(B1) For an ideal or isentropic compression, $\mathrm{d} s_{i r r}=0$, so $C_{p} \cdot \mathrm{d}\left(\ln T_{t}\right)=R \cdot \mathrm{d}\left(\ln p_{t}\right)$, which means the total pressure increases as energy is injected, and the total pressure decreases as energy is extracted;

(B2) For a real compression, $\mathrm{d} s_{i r r}=C_{p} \cdot \mathrm{d}\left(\ln T_{t}\right)-$ $R \cdot \mathrm{d}\left(\ln p_{t}\right)>0$, which means more energy than what is needed for the ideal compression must be injected at the same total pressure increment, and less energy will be extracted at the same decrease in total pressure;

(B3) For an adiabatic compression, $\mathrm{d} \ln \left(T_{t}\right)=0$, so $\mathrm{d} s_{i r r}=-R \cdot d \ln \left(p_{t}\right)$, which means the decrease in total pressure will be caused by the increase in entropy for process losses.

\section{DEMONSTRATION OF COMPRESSION REQUIRE- MENTS FOR INJECTION OR EXTRACTION OF EN- ERGY}

\section{Compression Required for Turbojets}

The temperature at the turbine inlet must be kept within a permissible range for the safe operation of a turbojet engine. Table 1 lists the specifications of some 3rd-generation turbofan engines characterized with $T_{t 3}$ in the range of $1600 \sim 1$ $800 \mathrm{~K}$. Corresponding to $T_{t 3}$, factor $\pi_{o p}$ is $20.1 \sim 21.4$ at sea level, $31.4 \sim 33.5$ at an altitude of $10 \mathrm{~km}$ and $33.1 \sim 35.3$ at an altitude of $20 \mathrm{~km}$ respectively.

It can be seen from Fig. (2) that for the applications listed in Table $1 \pi$ is always located within $\pi_{o p}$ range when $M a_{1}$ $=0.5 \sim 3$, except M53-P2 engine of Mirage 2000 aircraft. It can also be seen that $\pi$ is always greater than $p_{t 1} / p_{1}$. In

\begin{tabular}{|c|c|c|c|c|c|c|}
\hline Country & Engine Type & Aircraft Application & $\pi$ & $T_{t 3} / \mathbf{K}$ & $\underset{\left(v_{1}\right) / \mathbf{k m} \cdot \mathbf{h}^{-1}}{\text { Aircraft Max }}$ & Aircraft Max $\left(M a_{1}\right)$ \\
\hline \multirow{4}{*}{ USA } & F100-PW-220 & F-15 & 32 & 1643 & - & 2.5 \\
\hline & F110-GE-100 & F-16 & $29.9 \sim 30.4$ & 1643 & - & 2 \\
\hline & F404-F1D2 & F117 & 26 & 1643 & - & 0.92 \\
\hline & F110-GE-129 & - & 32 & 1728 & - & - \\
\hline \multirow{3}{*}{ Russia or USSR } & AL-31F & Su-27 & 23 & 1650 & 2430 & - \\
\hline & RD-133 & MIG-29 & 21 & 1536 & 2450 & - \\
\hline & D-30F6 & MIG-31 & 21 & 1660 & 3000 & - \\
\hline China PRC & Taihang growth versions & - & - & 1800 & - & - \\
\hline
\end{tabular}

Table 1. Specifications of Turbojet Engine Applications 
order to obtain $\pi$, the degree of the compression required for turbojets is formulated as inequality $p_{t 2} / p_{t 1}>1$.

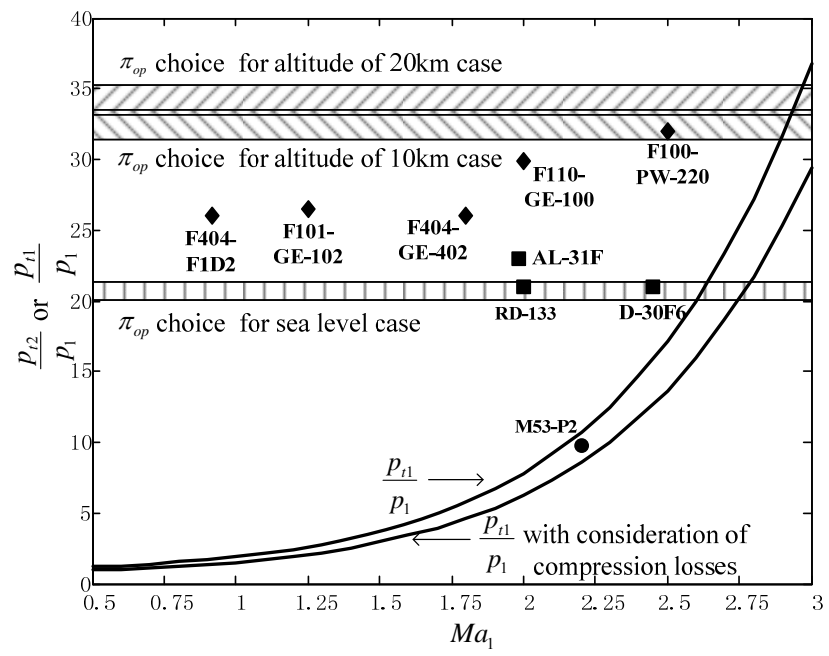

Fig. (2). Relationship between pressure ratio and flight Mach number for turbojet applications.

According to the indication (B1) of equation 3, the compression required can be obtained by the implementing the increase in total pressure induced by the injection of energy. The injection of energy is achieved in turbojet engines by a compressor in which the incoming flow is compressed simultaneously when energy is injected into the flow field, and the energy for the compressor comes from the energy extracted from a turbine. It can also be seen from Fig. 2 that if compression loss is considered, more energy is needed by the compressor design.

\section{Compression Required for Ramjets/Scramjets}

For a ramjet/scramjet engine, the determination of the total temperature at the combustor exit requires a combination of elaborate computations and experienced judgments, because such a temperature depends on many interrelated variables such as engine configuration, combustion detail, heat transfer and cooling method. Although there are many considerations, $T_{t 3}$ must be kept within a permissible range for an acceptable engine design. The $T_{t 3}$ range used in this paper is $2500 \sim 8500 \mathrm{~K}$. (Heiser AIAA ES 1994) [20] The corresponding $\pi_{o p}$ is $64.6 \sim 684.0$ at sea level, $105.6 \sim$ 1118.8 at an altitude of $10 \mathrm{~km}$ and $111.9 \sim 1185.5$ at an altitude of $20 \mathrm{~km}$ respectively.

The military nature of the applications of the ramjet/scramjet makes it difficult to have access to the detailed descriptions of the ramjet/scramjet. Data concerning the specific compression issues in this paper come from the published information about compression configurations and working conditions. Listed in Table $\mathbf{2}$ are some compression details, as the results of CFD analysis done with Fluent tools. (Kuranov JSR 2003) [16] (Rozario AIAA 2007) [21] (Emami NASA 1995 ) [22] (Patrick JPP 1996) [23] (Ding AIAA 2001) [24] (Rodriguez JPP 2003) [25] (Voland AIAA 1999) [26] (Ault JPP 1994) [27] (Van JPP 1996) [28] (Chan JSR 1995) [29]

It can be seen from Fig. 3 that $\pi$ is always less than $p_{t 1} / p_{1}$ in the range of $M a_{1}=3 \sim 9$. In order to obtain $\pi$, the degree of the compression required for ramjet/scramjet engines is formulated as inequality $p_{t 2} / p_{t 1}<1$.

In ramjet/scramjet engines, incoming flow is compressed by adiabatic shock interactions without the injection or extraction of energy, and the decrease in total pressure is caused by the increase in entropy induced by losses in the compression process. As shown in Fig. (3) that $\pi$ are located within $\pi_{o p}$ range when $M a_{1}=3 \sim 9$, the decrease in total pressure resulting from adiabatic shock interactions provides the compression required for the ramjet/scramjet applications in this speed range. Moreover, it can be seen from Fig. (4) that adiabatic shock interactions provide ram-

Table 2. Compression Details of Ramjet/Scramjet Engines

\begin{tabular}{|c|c|c|c|c|c|c|c|}
\hline Data Label & $M a_{1}$ & $p_{1} / \mathbf{P a}$ & $T_{1} / \mathrm{K}$ & $p_{t 1} / p_{1}$ & $2 / p_{t 1}\left(\times 10^{-2}\right)$ & $p_{t 2} / p_{1}$ & Data Source \\
\hline 1 & 4 & 9084.3 & 66.1 & 152.2 & 63.8 & 97.0 & Ref. $[21,22]$ \\
\hline 2 & 4 & 8960 & 170 & 152.2 & 73.6 & 112.1 & Ref. [23] \\
\hline 3 & 4 & 8003.1 & 73.1 & 152.2 & 56.4 & 85.8 & \multirow{3}{*}{ Ref. [24] } \\
\hline 4 & 4 & 8003.1 & 76.0 & 152.2 & 58.2 & 88.6 & \\
\hline 5 & 4 & 8003.1 & 76.0 & 152.2 & 64.4 & 97.9 & \\
\hline 6 & 6.4 & 3968 & 203.5 & 2369.0 & 30.6 & 725.2 & Ref. $[25,26]$ \\
\hline 7 & 9.9 & 634.3 & 98.6 & 39905 & 8.97 & 3578.0 & \multirow{4}{*}{ Ref. $[27,28]$} \\
\hline 8 & 10.2 & 234.4 & 48.7 & 49488 & 7.6 & 3766.3 & \\
\hline 9 & 10.4 & 606.8 & 48.7 & 55446 & 8.4 & 4646.9 & \\
\hline 10 & 13.1 & 110.3 & 48.1 & 271818 & 3.3 & 8869.9 & \\
\hline 11 & 8 & 157 & 447 & 9844.3 & 6.5 & 637.9 & Ref. [29] \\
\hline 12 & 10 & 1200 & 226 & 42900 & 10.4 & 4497.6 & Ref. [16] \\
\hline
\end{tabular}


jet/scramjet engines with acceptable changes in total pressure in the compression processes when $M a_{1}=3 \sim 9$, which are also significantly demanded for the design of ramjet/scramjet engines.

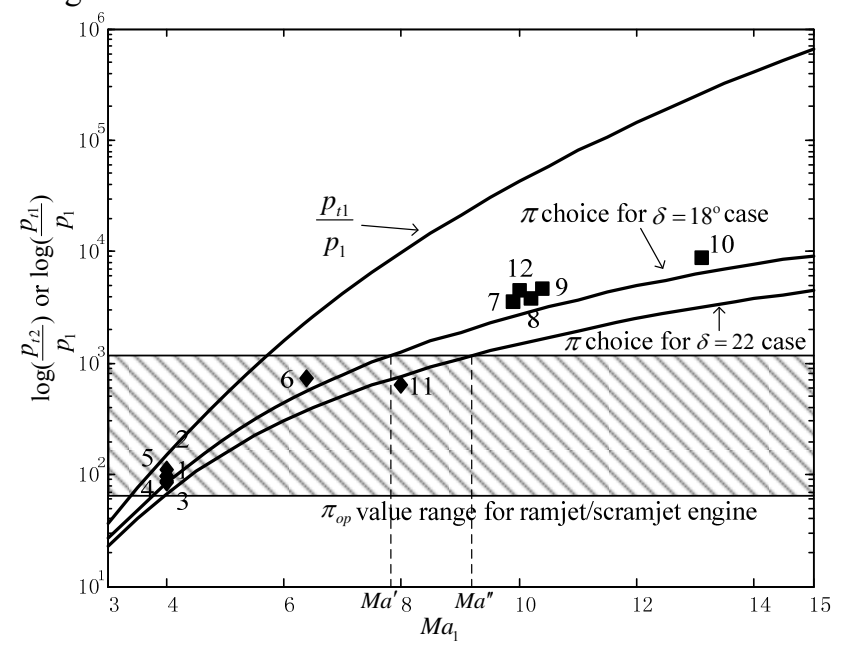

Fig. (3). Relationship between pressure ratio and flight Mach number for ramjet/scramjet applications.

\section{Compression Required for Ramjets with Energy-Bypass}

It can be seen from Fig. (3) that when $M a_{1}>9, \pi$ is always less than $p_{t 1} / p_{1}$, and $\pi$ locates beyond $\pi_{o p}$ range. This means that although the decrease in total pressure is caused by the increase in entropy induced by shock compression losses, the decrease in total pressure can not provide the compression required for applications with $M a_{1}>9$. In order to make $\pi$ approach $\pi_{o p}$, more decrease in total pressure than that listed in Table $\mathbf{2}$ is needed. Thus when $M a_{1}>9$, inequality $p_{t 2} / p_{t 1}<1$ can be explained as a deep decrease in total pressure.

This compression required can be simply provided by a deep compression. More losses in the deep compression process result in more decrease in total pressure. More decrease in total pressure provide the compression required so that the deep compression makes $\pi$ approach $\pi_{o p}$. Simultaneously the corresponding $p_{t 2} / p_{t 1}$ of the deep compression will be the order of magnitude of $10^{-1}$ or even less, as shown in Fig. (4). For adiabatic shock interactions, such $p_{t 2} / p_{t 1}$ results in a significant entropy increase which is detrimental to engine performance. Therefore, the deep compression can not provide a comprehensive solution to the airflow compression when $M a_{1}>9$.

It can be seen from the above analyses that both the deep decrease in total pressure and an acceptable process entropy increment are needed when $M a_{1}>9$. In this case, the compression required can be re-explained as the combination of the deep decrease in total pressure and the acceptable process entropy increment. Based on equation 3, the extraction of energy and the increase in entropy can all cause the decrease in total pressure. Thus the compression required for applications with $M a_{1}>9$ can be provided in a way that the decrease in total pressure is mainly caused by the extraction of energy, but not by the increase in entropy. This means that the deep decrease in total pressure can be mainly caused by the extraction of energy, and most importantly the process entropy increment would also be kept in a permissible level.

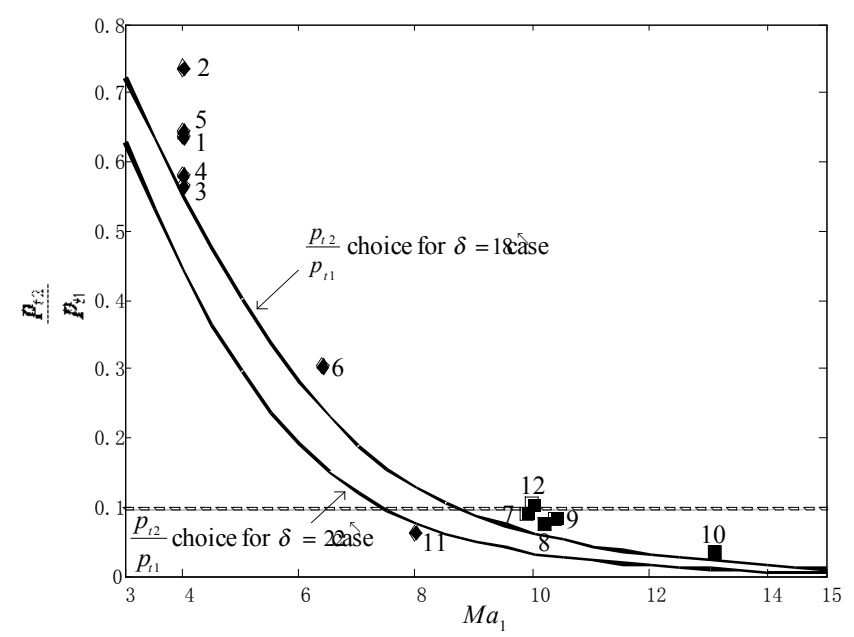

Fig. (4). Relationship between total pressure ratio and flight Mach number for ramjet/scramjet applications.

The ramjet with energy-bypass, which has the combination of the adiabatic shock compression process and the energy extraction process upstream of the combustor, can provide the compression required for applications with $M a_{1}>9$. Moreover, the return of the extracted energy downstream of the combustor is achieved for the efficient use of energy, since the extracted energy is sourced from the movement of engine/aircraft.

It should be noted that $M a_{1}=9$ is used here as the terminal of the application range. In fact, the determination of this value requires a combination of detailed computations and comparative analyses of engine performance for with or without energy-bypass. The use of this specific value in this paper is only because as shown in Fig. (3), $\pi$ locates in $\pi_{o p}$ range when $M a_{1}=3 \sim 9$ and $\pi$ locates beyond $\pi_{o p}$ range when $M a_{1}>9$. It is assumed for intuitive demonstration that the incoming flow is compressed through four ramps with equal shock angles $\delta$, and then processed with or without the energy extraction process under different conditions. It can be seen from Fig. (3) that the achievement of the extraction of energy would be desirable for the compression required when $M a_{1}$ is greater than a specific value in the different $\delta$ case.

\section{DEVELOPMENT TREND OF COMPRESSION PROCESSES IN AERO-ENGINE THERMAL CYCLES}

As discussed above, the injection of energy is required for the turbojet compression; the method of adiabatic shock interactions is desirable for the ramjet/scramjet compression; and the extraction of energy is needed in the ramjet with energy-bypass. 
Thus, with the increase in the flight Mach number, the compression processes of aero-engine thermal cycles consist of the compression with energy injection in the case of low speed ranges, the adiabatic compression in the case of intermediate speed ranges, and the compression with energy extraction in the case of high speed ranges. The injection or extraction of energy in the compression process aims at the realization of the engine performance required for applications in different speed ranges.

It should be noted that when extraction of energy is required in the compression process for applications with $M a_{1}$ $>9$, ramjet with energy-bypass is one of the possible solutions, and a new type of engine can be developed when a new way of energy extraction is available for hypersonic applications.

\section{DISCUSSION}

There are many data sources about turbojet applications in the open literature as well as very limited descriptions about ramjet/scramjet applications. Due to commercial competitions or military secrets, it is no doubt that there are differences between the data adopted in this paper and the real engine attributes. We cannot guarantee the accuracy of public data. However, these data differences have little effect on the viewpoint of this paper, because this work objective is to analyze the public data and demonstrate the general trends, but not to discuss any particular design. Furthermore, these data differences may cause the imprecise determination of the terminal of those engine application ranges.

The precise determination of the terminal of engine application ranges is not rational in real life, since there are always common application ranges for two types of engines. Just like the range of $M a_{1}=1.5 \sim 3$ as the common range of turbojet and ramjet/scramjet applications, there must also be a common flight speed range, in which ramjet/scramjet with or without energy-bypass are developed for different applications. Furthermore, just like the range of $M a_{1}<3$ as the dominant range of turbojet applications, there must also be a specific range for the applications of ramjet with energybypass, which can be expressed as $M a_{1}>9$ or a faster speed range. In this paper, the uses of the specific values as the terminal of those engine application ranges are only due to the data locations shown in Figs. (2-4).

\section{CONCLUSIONS}

The evolution of the compression processes in aeroengine thermal cycles is reviewed. The theoretical analyses about the compression processes show that the injection or extraction of energy in the compression process heavily depends on the aero-engine performance required for applications in different speed ranges. The injection of energy in the compression process is required for applications in low speed ranges, for example, the injection of energy in turbojet compressors; the method of adiabatic compression is desirable for applications in intermediate speed ranges, for example, shock interactions for ramjets/scramjets; and the extraction of energy is needed for applications in high speed ranges, for example, the extraction of energy in ramjets with energy-bypass.

\section{ACKNOWLEDGEMENTS}

This work is funded by National Natural Science Foundation of China under contract No. 50306003, Hi-Tech Research and Development Program of China under contract No. 2002AA722105, and Aeronautics Technology Support Foundation under contract No. Hagong07. The appreciation will also be given to Dr. E. G. Sheikin of hypersonic systems research institute of Russia for the academic communication, and professor Y. Jialu of Harbin institute of technology of China PRC for the instructions.

\section{REFERENCES}

[1] D.W. Riggins, "Analysis of the magneto-hydrodynamic energy bypass engine for high-speed airbreathing propulsion" J. Prop. Power, vol. 20(5), pp. 779-792, 2004.

[2] V.L. Fraishtadt, A.L. Kuranov, and E.G. Sheikin, "Use of MHD systems in hypersonic aircraft", Tech. Phys., vol. 43(11), pp. 1309 1313, 1998.

[3] E.G. Sheikin, "Analytical solution of the system of MHD equations in the quasi-one-dimensional approximation for regimes in which the flow parameters vary monotonically along the channel", Tech. Phys., vol. 37(12), pp. 1133-1136, 1992.

[4] E.G. Sheikin, "Analytical solution of a system of quasi-onedimensional MHD equations for flow whose parameters vary monotonically along the channel length. Optimum choice of parameters for an MHD generator", Tech. Phys., pp. 38(9), 741-746, 1993

[5] A.L. Kuranov, A.V. Korabelnicov, V.V. Kuchinskiy, and E.G. Sheikin, "Fundamental techniques of the AJAX concept, modern state of research", AIAA Paper, 2001-1915.

[6] E.P. Gurijanov, and P.T. Harsha, "AJAX: new direction in hypersonic technology", AIAA Paper, 1996- 4609.

[7] J.F. Tang, W. Bao, and D.R. Yu, "A new manner for energy reintroduction in AJAX”, AIAA Paper, 2006-8101.

[8] J.F. Tang, W. Bao, and D.R. Yu, "The theoretical treatment of comparison of performance between two scramjet engines with energy-bypass based on exergy method", AIAA Paper, 2007-1381.

[9] C. Park, D.W. Bogdanoff, and U.B. Mehta, "Theoretical performance of frictionless magneto-hydrodynamic-bypass scramjets", $J$. Prop. Power, vol. 17(3), pp. 591-598, 2001.

[10] O.M. Sergey, N.S. Mikhail, B.M. Richard, and J.L. Ronald, "Electron beam generated plasmas in hypersonic MHD channels", AIAA J., vol. 39(6), pp. 1127-1138, 2001.

[11] O.M. Sergey, N.S. Mikhail, and B.M. Richard, "MHD power extraction from cold hypersonic air flows with external ionizers" $J$. Prop. Power, vol. 18(2), pp. 424-441, 2002.

[12] C. Park, D.W. Bogdanoff, and U.B. Mehta, "Theoretical performance of a magneto-hydrodynamic-bypass scramjet engine with nonequilibrium ionization", J. Prop. Power, vol. 19(4), pp. 529537, 2003.

[13] R.J. Litchford, J.W. Cole, V.A. Bityurin, and T.L. John, "Thermodynamic cycle analysis of magneto-hydrodynamic-bypass hypersonic airbreathing engines", J. Prop. Power, vol. 17(2), pp. 477480, 2001.

[14] C. Park, U.B. Mehta, and D.W. Bogdanoff, "Magnetohydrodynamics energy bypass scramjet performance with real gas effects", J. Prop. Power, vol. 17(5), pp. 1049-1057, 2001.

[15] B.M. Burakhanov, A.P. Likhachev, and S.A. Medin, "Advancement of scramjet magneto-hydrodynamic concept", J. Prop. Power, vol. 17(6), pp. 1247-1252, 2001.

[16] A.L. Kuranov, and E.G. Sheikin, "Magneto-hydrodynamic control on hypersonic aircraft under AJAX concept", J. Spacecra. Rocke., vol. 40(2), pp. 174-182, 2003.

[17] W.H. Heiser, and D.T. Pratt, "Comment on analysis of the magneto-hydrodynamic energy bypass engine for high-speed airbreathing propulsion", J. Prop. Power, vol. 21(6), p. 1140, 2005.

[18] E.G. Sheikin, and A.L. Kuranov, "MHD control in hypersonic aircraft”, AIAA Paper, 2005-1335.

[19] A. Bejan, "Advanced engineering thermodynamics", Wiley, New York 1988.

[20] W.H. Heiser, D.T. Pratt, D.H. Daley, and U.B. Mehta, "Hypersonic airbreathing propulsion", AIAA education series 1994. 
[21] D. Rozario, and Z. Zouaoui, "Computational fluid dynamic analysis of scramjet inlet", AIAA Paper, 2007-30.

[22] S. Emami, and C.A. Trexler, "Experimental investigation of inletcombustor isolators for a dual-mode scramjet at a Mach number of 4", NASA Technical Paper, 1995-3502.

[23] E.R. Patrick, E. Saied, and A.T. Carl, "Unsteady pressure behavior in a ramjet/scramjet inlet", J. Prop. Power, vol. 12(3), pp. 486-493, 1996.

[24] M. Ding, L. Hua, and F. Xiao, "Two-dimensional viscous simulation of a inlet-isolator flows at mach number 4", AIAA Paper, 2001- 3883 .

[25] C.G. Rodriguez, "Computational fluid dynamics analysis of the central institute of aviation motors/NASA scramjet", J. Prop. Power, vol. 19(4), pp. 547-554, 2003.
[26] R.T. Voland, A.H. Auslender, M.K. Smart, A.S. Roudakov, V.L. Semenov, and V. Kopchenov, "CIAM/NASA mach 6.5 scramjet flight and ground test", AIAA Paper, 1999-4848.

[27] D.A. Ault, and D.M. Van Wie, "Experimental and computational results for the external flowfield of a scramjet inlet", J. Prop. Power, vol. 10(4), pp. 533-539, 1994.

[28] D.M. Van Wie, and D.A. Aultt, "Internal flowfield characteristics of a scramjet inlet at mach 10", J. Prop. Power, vol. 12(1), pp. 158164, 1196.

[29] H.C. Chan, C.K. Suk, J.D.W. Kenneth, and T.N. Henry, "Numerical analysis of hypersonic low-density scramjet inlet flow", $J$. Spacecra. Rocke., vol. 32(1), pp. 60-66, 1995. 